\title{
Bultmann Handbuch
}

\author{
Hrsg. v. Christof Landmesser
}

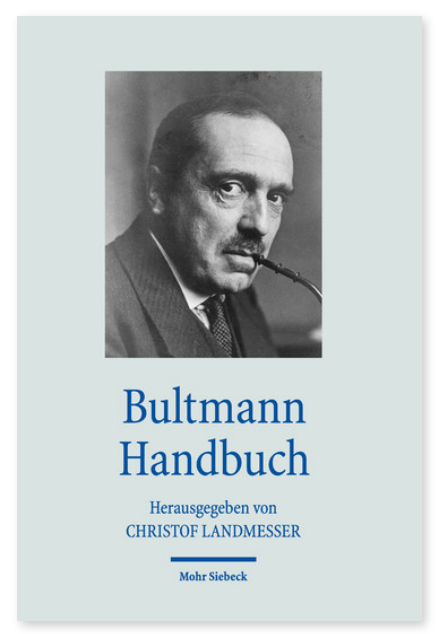

2017. XI, 546 Seiten. HdTh

SBN 978-3-16-154903-8

DOI 10.1628/978-3-16-154903-8

eBook PDF 149,00€

ISBN 978-3-16-151687-0

fadengeheftete Broschur 49,00€

ISBN 978-3-16-151688-7

Leinen $149,00 €$

Rudolf Bultmann (1884-1976) prägte durch seinen hermeneutischen Ansatz die exegetischen und systematischtheologischen sowie kirchlichen Diskurse des 20. Jahrhunderts wesentlich mit. Als Mitbegründer der formgeschichtlichen Schule und früher Vertreter der Dialektischen Theologie setzte er sich in den 1920er Jahren kritisch mit Positionen der liberalen Theologie auseinander und rückte die hermeneutische Frage nach den Verstehensbedingungen der biblischen Texte sowie deren Bedeutung für die Leserinnen und Leser in der Moderne in den Fokus seiner wissenschaftlichen Arbeit. Seine Theologie entwickelte Bultmann im Gespräch und in der Auseinandersetzung; so pflegte er einen intensiven Austausch mit Kolleginnen und Kollegen auch anderer wissenschaftlicher Disziplinen, mit Studentinnen und Studenten, mit Pfarrerinnen und Pfarrern.

Dieses Handbuch bietet neben einem ersten Orientierungsabschnitt über Bultmanns Werke und den gegenwärtigen Forschungsstand, in einem zweiten Abschnitt einen Zugang zur Person. Darin werden die Biographie, die theologischen Prägungen, die Beziehungen zu wichtigen Gesprächspartnern und seine politisch-gesellschaftlichen Kontexte in den Blick genommen. Eine Beschäftigung mit dem Werk Bultmanns findet im dritten Abschnitt statt. In diesem Abschnitt werden die vielfältigen Gattungen und Themen seines Fuvres behandelt sowie die sein Gesamtwerk prägenden Strukturen. Schließlich wird die Wirkung und Rezeption seiner Theologie insbesondere im deutschsprachigen Raum dargestellt und diskutiert. Das Handbuch eignet sich für eine erste Orientierung in der Beschäftigung mit Bultmann; es ist darüber hinaus auch ein Nachschlagewerk für Fachleute und Bultmann-Kenner.

Inhaltsübersicht

\section{A. Orientierung}

I. Johannes Beck: Bultmanns Werke: Einzelausgaben, Aufsatzbände, Editionen

II. Johannes Beck: Bultmannforschung: Hilfsmittel, Institutionen und gegenwärtige Forschung

B. Person

I. Konrad Hammann: Biographisches Umfeld und Vita

\section{Traditionen}

Christine Axt-Piscalar: Augustin, Luther und das Luthertum - Claudia Welz: Kierkegaard - Johannes Beck: Schleiermacher, Dilthey - Christina Kuß: Historisch-kritische Tradition - Christoph Herbst: Religionsgeschichtliche Schule und "Liberale Theologie«

\section{Beziehungen}

Alexander Heit: Bultmann und Martin Rade - Ernst Baasland: Bultmann und Hermann Gunkel - Alexander Heit: Bultmann und Friedrich Gogarten - Alexander Heit: Bultmann, Karl Barth und die Dialektische Theologie - Andreas Großmann: Bultmann und Martin Heidegger - Andreas Großmann: Bultmann und Karl Jaspers - Wolfram Kinzig: Bultmann und Hans von Soden - Arnulf von Scheliha: Bultmann und Emanuel Hirsch - Andreas Großmann: Bultmann und Rudolf Otto - Andreas Großmann: Bultmann und Marburger Kollegen - Christina Kuß: Bultmann und Heinrich Schlier - Konrad Hammann: Bultmann und Hans Jonas - Friederike Portenhauser: Bultmann und Ernst Käsemann - Albrecht Beutel: Bultmann und Gerhard Ebeling - Oliver Pilnei: Bultmann und Ernst Fuchs - Werner Zager: Bultmann, Günther Bornkamm, Herbert Braun, Hans Conzelmann, Walter Schmithals - Andreas Großmann: Bultmann, Hans-Georg Gadamer, Gerhard Krüger, Karl Löwith, Wilhelm Anz

IV. Politisch-gesellschaftliche Beziehungen

Hans-Peter Großhans: Bultmann und die Kirche - Christian Polke: Bultmann und die Politik - Konrad Hammann: Bultmann und das Judentum - Thomas Dörken-Kucharz: Bultmann und die Kultur

\section{Werk I. Gattungen}

Christina Kuß: Monographien und Kommentare - Johannes Beck: Vorträge und Aufsätze - Matthias Dreher: Rezensionen und Forschungsberichte - Martin Bauspieß: Lexikonartikel - Eberhard Hauschildt: Predigten - Christina Kuß/Friederike Portenhauser: Briefe

II. Strukturen

Elisabeth Gräb-Schmidt: Sünde und Rechtfertigung - Hans Weder: Glauben und Verstehen - Christof Landmesser: Selbstverständnis und Weltverständnis - Karin Scheiber: Freiheit und Gehorsam, Freiheit und Bindung

\section{Themen}

Andreas Lindemann: Religionsgeschichtliches Umfeld des Neuen Testaments (Hellenismus, Judentum, Urchristentum) - Enno Edzard Popkes: Gnosis - Paul-Gerhard Klumbies: Die synoptische Überlieferung - Michael Theobald: Jesus - Christof Landmesser: Paulus - Michael Labahn: Johannes/Johanneische Theologie - Christof Landmesser: Theologie des Neuen Testaments - Manfred Oeming: Bultmann und das Alte Testament - Birgit Weyel: Religion - Martin Bauspieß: Geschichte Folkart Wittekind: Eschatologie - Christof Landmesser: Anthropologie - Christoph Seibert: Glaube - Elisabeth Gräb-Schmidt: Ethik - Martin Wendte: Der Begriff der Offenbarung - Ulrich H. J. Körtner: Wort-Gottes-Theologie - Ulrich H. J. Körtner: Enzyklopädische Theologie - Christof Landmesser: Hermeneutik und existentiale Interpretation - Paul-Gerhard Klumbies: Mythos und Entmythologisierung - Martin Bauspieß: Frühkirchliche Entwicklungen - Hartmut Rosenau: Theologie und 
Philosophie

D. Wirkung und Rezeption

I. Andreas Lindemann: Bultmannschule

II. Stephan Schaede: Entmythologisierungsdebatte

III. Michae/ Theobald: Bultmannrezeption in der Jesusforschung

IV. Andreas Lindemann: Bultmannrezeption in der Paulusforschung

V. Udo Schnelle: Bultmannrezeption in der Johannesforschung

VI. Enno Edzard Popkes/Hartmut Rosenau: Bultmannrezeption in der Systematischen Theologie und in der neueren religionsgeschichtlichen Debatte

VII. Stephan Grätzel: Bultmannrezeption in der Philosophie

VIII. Francis Watson: Bultmannrezeption im englischsprachigen Raum

IX. Ernst Baasland: Bultmannrezeption in Skandinavien

Christof Landmesser Geboren 1959; 1998 Promotion; 2000 Habilitation; 2003-06 Professor für Neues Testament an der Johannes Gutenberg-Universität in Mainz; seit 2006 Professor für Neues Testament an der Eberhard Karls Universität Tübingen.

Jetzt bestellen:

https://mohrsiebeck.com/buch/bultmann-handbuch-9783161549038?no_cache=1

order@mohrsiebeck.com

Telefon: +49 (0)7071-923-17

Telefax: +49(0)7071-51104 